Check for updates

Cite this: RSC Adv., 2019, 9, 1387

Received 27th December 2018

Accepted 3rd January 2019

DOI: $10.1039 / \mathrm{c} 8 \mathrm{ra10601k}$

rsc.li/rsc-advances

\section{Strong interfacial interactions induced a large reduction in lateral thermal conductivity of transition-metal dichalcogenide superlattices}

\begin{abstract}
Wenjie Zhang, Jia-Yue Yang (D)* and Linhua Liu*
van der Waals heterostructures formed by vertically stacking two-dimensional (2D) crystals can not only harness the already fascinating properties of their constituent monolayers but also extend them due to the coupled interlayer interactions. With their emerged interlayer and intralayer excitons, 2D transition metal dichalcogenides (TDMC) crystals and their heterostructures have drawn growing attention for the applications of nanoelectronics, optoelectronics and nanophotonics. Yet, there are few studies on how interlayer and interfacial interactions influence the thermal transport in TDMC heterostructures which is critical for heat management. In this work, we investigate the lateral and out-of-the-plane thermal conductivity $(\kappa)$ of four prototype TDMC heterostructures (bilayer $\mathrm{MoS}_{2}$ and $\mathrm{WS}_{2}$, heterobilayer $\mathrm{MoS}_{2} / \mathrm{WS}_{2}$ and superlattice $\mathrm{MoS}_{2} / \mathrm{WS}_{2}$ ) by solving the phonon Boltzmann transport equation from first-principles. The calculated room-temperature lateral $\kappa$ of bilayer $\mathrm{MoS}_{2}$ and $\mathrm{WS}_{2}$ are $61.13 \mathrm{~W} \mathrm{~m}^{-1} \mathrm{~K}^{-1}$ and $87.52 \mathrm{~W} \mathrm{~m}^{-1} \mathrm{~K}^{-1}$, respectively, in reasonable agreement with literature experiments. The weak interlayer interactions in the heterobilayer $\mathrm{MoS}_{2} / \mathrm{WS}_{2}$ help preserve the high lateral thermal transport $\left(70.01 \mathrm{~W} \mathrm{~m}^{-1} \mathrm{~K}^{-1}\right.$ ) of its constituent monolayer. In the superlattice $\mathrm{MoS}_{2} / \mathrm{WS}_{2}$, there exist strong interlayer and interfacial interactions between the alternating $\mathrm{MoS}_{2}$ and $\mathrm{WS}_{2}$ monolayer which reduce the lateral $\kappa$ to be $7.22 \mathrm{~W} \mathrm{~m}^{-1}$ $\mathrm{K}^{-1}$ by a factor of 10 . The greatly reduced lateral $\kappa$ of the superlattice mainly arises from the low phonon relaxation time, which indicates the existence of strong interfacial anharmonic phonon scattering. This work aims at uncovering the physics of emerged thermal transport properties in TDMC heterostructures and helps advance their applications in heat management among nanoelectronics and optoelectronics.
\end{abstract}

\section{Introduction}

With their unique combination of direct bandgaps, atomicscale thickness, strong light-matter interactions and spinorbital coupling, and favorable mechanical properties, twodimensional (2D) transition metal dichalcogenides (TMDC) have become the emerging class of materials for applications in next-generation nanoelectronics, spintronics and nanophotonics. $^{1-7}$ Considerable efforts have enabled the robust growth of heterostructures and superlattices from 2D TDMCs with predetermined attributes and functionalities. ${ }^{8-12}$ Held together by van der Waals forces, different TDMC layers with diverse properties vertically stack on each other and their quantum states interact and couple to one another, which could generate fascinating electrical, optical and thermal properties in $2 \mathrm{D}$ heterostructures and superlattices. ${ }^{13-16}$

Among those properties, exploring thermal transport in the 2D TDMC heterostructures and superlattices is not only of fundamental physics interest but also crucial to advance heat management for their applications in nanoelectronics and

School of Energy and Power Engineering, Shandong University, Qingdao 266237, China.E-mail: jy_yang@sdu.edu.cn; liulinhua@sdu.edu.cn optoelectronics. Using a refined optothermal Raman technique, Zhang and co-workers ${ }^{17}$ measured the lateral thermal conductivity $(\kappa)$ and interfacial thermal conductance of suspended single and bilayer $\mathrm{MoS}_{2}$ and $\mathrm{MoSe}_{2}$ at room temperature. By analyzing the temperature and excitation dependences of active Raman modes, Peimyoo et al. ${ }^{18}$ determined the lateral $\kappa$ of suspended single and bilayer $\mathrm{WS}_{2}$. Using classical molecular dynamics, Zhang and coworkers ${ }^{19}$ calculated the lateral $\kappa$ of heterobilayer $\mathrm{MoS}_{2} / \mathrm{MoSe}_{2}$ and showed that it preserved the high thermal conductivity of most 2D TDMC materials. Moreover, the interfacial interactions had little influence on the lateral thermal performance. Those studies greatly advance the understanding of thermal transport in $2 \mathrm{D}$ TDMC and the related heterostructures. Yet to modulate and engineer thermal transport in the 2D TDMC heterostructures with a higher precision, it requires a sound knowledge on phonon transport in 2D TDMCs and how the vertically stacking TDMCs layers influence the quantum behaviors of phonons.

In this work, we choose four prototype TDMCs, bilayer $\mathrm{MoS}_{2}$ and $\mathrm{WS}_{2}$, heterobilayer $\mathrm{MoS}_{2} / \mathrm{WS}_{2}$ and superlattice $\mathrm{MoS}_{2} / \mathrm{WS}_{2}$, and investigate their lateral and out-of-the-plane $\kappa$ using firstprinciples calculations without empirical parameters. By analyzing anharmonic lattice dynamics coupled with the Boltzmann transport theory, the calculated lateral $\kappa$ of bilayer 
$\mathrm{MoS}_{2}$ and $\mathrm{WS}_{2}$ agree with previous experiments. In comparison, the lateral $\kappa$ of heterobilayer $\mathrm{MoS}_{2} / \mathrm{WS}_{2}$ preserves the high values of bilayer $\mathrm{MoS}_{2}$ and $\mathrm{WS}_{2}$, and the interlayer van der Waals forces has little influence on it. By comparing heterobilayer and superlattice $\mathrm{MoS}_{2} / \mathrm{WS}_{2}$, it shows that the strong interfacial interactions in the superlattice greatly affect the lateral $\kappa$ of heterobilayer and reduce it by a factor of 10 .

\section{Computational methodology}

The 2D TDMC semiconductors exhibit $2 \mathrm{H}$ symmetry with the general formula $\mathrm{MX}_{2}$, where $\mathrm{M}=\mathrm{Mo}, \mathrm{W} ; \mathrm{X}=\mathrm{S}$, Se and Te. In Fig. 1, the lattice structures of bilayer $\mathrm{MoS}_{2}$ and $\mathrm{WS}_{2}$, heterobilayer $\mathrm{MoS}_{2} / \mathrm{WS}_{2}$ and superlattice $\mathrm{MoS}_{2} / \mathrm{WS}_{2}$ are presented. After structure optimization, the thickness of bilayer $\mathrm{MoS}_{2}$ and $\mathrm{WS}_{2}$ and heterobilayer $\mathrm{MoS}_{2} / \mathrm{WS}_{2}$ is $0.9605 \mathrm{~nm}, 0.9633 \mathrm{~nm}$ and $0.9622 \mathrm{~nm}$, respectively. To avoid the interlayer van der Waals interaction, one $1 \mathrm{~nm}$-thick vacuum layer was introduced. The relaxed lattice parameter for the superlattice $\mathrm{MoS}_{2} / \mathrm{WS}_{2}$ was $a(b)=0.3281 \mathrm{~nm}$ and $c=1.2986 \mathrm{~nm}$. After performing the phonon dispersion relation calculations, the nonexistence of imaginary phonon modes indicates the dynamic stability of those TDMCs structures.

In 2D TDMC semiconductors, the thermal transport is dominated by the free carriers of phonons whose transport capacities are mainly limited by the intrinsic phonon-phonon interaction. ${ }^{\mathbf{2 0 - 2 2}}$ According to Boltzmann transport theory, $\kappa$ is determined by ${ }^{23,24}$

$$
\kappa=\frac{1}{N \Omega} \sum_{\boldsymbol{q} v} C_{\boldsymbol{q} v} v_{\boldsymbol{q} v}{ }^{2} \tau,
$$

where $N$ is the total number of $\boldsymbol{q}$ points in the first Brillouin zone, $\Omega$ is the volume of unit cell, $C_{\boldsymbol{q} v}, v_{\boldsymbol{q} v}$ and $\tau$ is the specific heat, group velocity and phonon relaxation time, respectively. Within the three-phonon scattering scheme, the key parameter $\tau$ determining thermal transport capability is given by ${ }^{25,26}$

$$
\begin{aligned}
1 / \tau= & \frac{2 \hbar \pi}{N} \sum_{\boldsymbol{q}^{\prime} v^{\prime} \boldsymbol{q}^{\prime \prime} v^{\prime \prime}}\left|V_{\boldsymbol{q} v \boldsymbol{q}^{\prime} v^{\prime} \boldsymbol{q}^{\prime \prime} v^{\prime \prime}}^{ \pm}\right|^{2}\left(n_{0}+1\right)\left(n_{0}^{\prime}+1 / 2 \pm 1 / 2\right) n_{0}^{\prime \prime} \delta\left(\omega_{\boldsymbol{q} v}\right. \\
& \left. \pm \omega_{\boldsymbol{q}^{\prime} v^{\prime}}-\omega_{\boldsymbol{q}^{\prime \prime} v^{\prime \prime}}\right)
\end{aligned}
$$

where $n_{0}$ is the Bose-Einstein distribution statistics of phonons and the delta function $\delta$ ensures energy conservation during phonon scattering process. The $V^{ \pm}$is the relevant three-phonon scattering matrix and determined by ${ }^{25,26}$

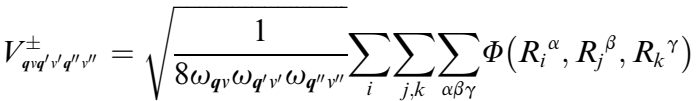

$$
\begin{aligned}
& \times \frac{\boldsymbol{e}_{\boldsymbol{q} v}{ }^{\alpha}(i) \boldsymbol{e}_{ \pm \boldsymbol{q}^{\prime} v^{v^{\prime}}}(j) \boldsymbol{e}_{-\boldsymbol{q}^{\prime \prime} v^{\prime \prime}} \gamma(k)}{\sqrt{M_{i} M_{j} M_{k}}},
\end{aligned}
$$

(a)

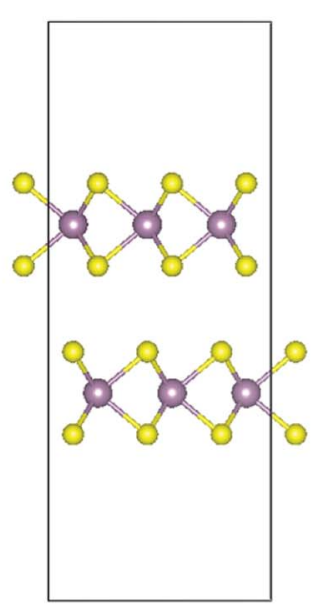

(c)

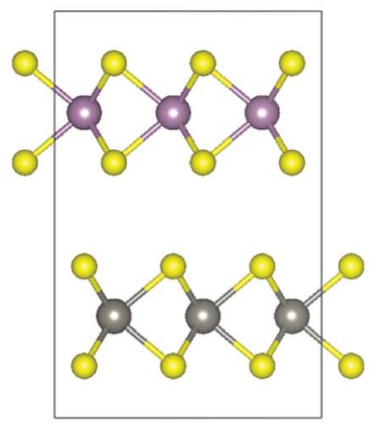

(b)

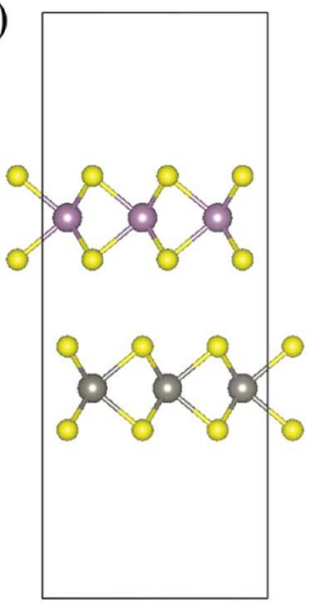

(d)

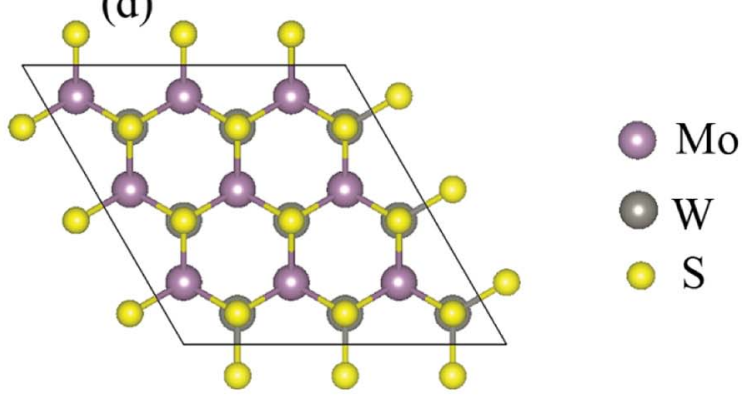

Fig. 1 The side view of bilayer (a) $\mathrm{MoS}_{2}$, (b) heterobilayer $\mathrm{MoS}_{2} / \mathrm{WS}_{2}$, (c) superlattice $\mathrm{MoS}_{2} / \mathrm{WS}_{2}$ and (d) top view of TDMC heterostructures. 
where $M$ is the corresponding mass, $R$ is the position for atoms $i, j$ and $k$ in the simulated cell, $\Phi$ is the third-order interatomic force constant (IFC), and $\boldsymbol{e}$ is the normalized eigenfunction and $\alpha, \beta$, and $\gamma$ denote the Cartesian coordinates. To calculate $V^{ \pm}$, it requires the harmonic interatomic force constants to obtain phonon dispersion relation and eigenvectors and third-order IFCs. To calculate the harmonic and third-order IFCs, we built a $5 \times 5 \times 1$ supercell containing 150 atoms and applied the finite-difference approach. ${ }^{27}$ During the calculation of thirdorder IFCs, the $8^{\text {th }}$ nearest neighbor was chosen and the cutoff radius was $0.65 \mathrm{~nm}$.

To calculate the harmonic and third-order IFCs, we employed the Vienna ab initio simulation package (VASP) ${ }^{28}$ which uses the projector augmented wave (PAW) method. ${ }^{29}$ The Perdew-Burke-Ernzerhof (PBE) ${ }^{\mathbf{3 0 , 3 1}}$ exchange-correlation functional was adopted and the vdW-DF2 functional ${ }^{32}$ was included to calculate the van der Waals forces. The Brillouin-zone integration was computed with the $12 \times 12 \times 1(12 \times 12 \times 12$ for superlattice) Monkhorst-Pack $k$-mesh ${ }^{33}$ and the cutoff energy was $600 \mathrm{eV}$. For the structural relaxation, the force convergence threshold was set as $0.1 \mu \mathrm{eV} \AA^{-1}$. After collecting the harmonic and third-order IFCs, the phonon relaxation time and thermal conductivity were computed with the ShengBTE package. ${ }^{25}$ The automatically generated $20 \times 20 \times 1(20 \times 20 \times 20$ for the superlattice) $\boldsymbol{q}$ grid was employed and the Gaussian smearing with breadth of 1 was applied.

\section{Results and discussions}

\section{Phonon dispersion relation}

Prior to computing thermal conductivity of TDMC heterostructures, it is essential to understand their quantum behaviors of phonons. In Fig. 2, the phonon dispersion relations and projected density of states (PDOS) of bilayer $\mathrm{MoS}_{2}$ and $\mathrm{WS}_{2}$, heterobilayer $\mathrm{MoS}_{2} / \mathrm{WS}_{2}$ and superlattice $\mathrm{MoS}_{2} / \mathrm{WS}_{2}$ are presented and compared. Since van der Waals (vdW) forces hold the TDMC layers together in the above heterostructures, the inclusion of vdW functional for the force calculations helps stabilize the bilayer, heterobilayer and superlattice heterostructures and eliminate the imaginary phonon modes. Due to the same $2 \mathrm{H}$ symmetry in lattice structure, there exists large similarity in the phonon dispersion relation of bilayer $\mathrm{MoS}_{2}$ and $\mathrm{WS}_{2}$ except that bilayer $\mathrm{MoS}_{2}$ has relatively higher phonon frequencies. That is because the mass of Mo (95.94 amu, $1 \mathrm{amu}$ $\left.=1.660539 \times 10^{-27} \mathrm{~kg}\right)$ is much lighter than that of $\mathrm{W}(183.84$ $\mathrm{amu}$ ). By vertically stacking one $\mathrm{MoS}_{2}$ layer on top of one $\mathrm{WS}_{2}$ layer, the newly formed $\mathrm{MoS}_{2} / \mathrm{WS}_{2}$ heterobilayer still keeps the $2 \mathrm{H}$ symmetry and its phonon dispersion keeps the similar shape of bilayer $\mathrm{MoS}_{2}$ and $\mathrm{WS}_{2}$. However, the phonon degeneracy is decreased and more phonon modes are generated, which is mainly caused by the coupled quantum states between $\mathrm{MoS}_{2}$ layer and $\mathrm{WS}_{2}$ layer. By periodically alternating the $\mathrm{MoS}_{2}$ and $\mathrm{WS}_{2}$ layer, the superlattice $\mathrm{MoS}_{2} / \mathrm{WS}_{2}$ is built. By comparing
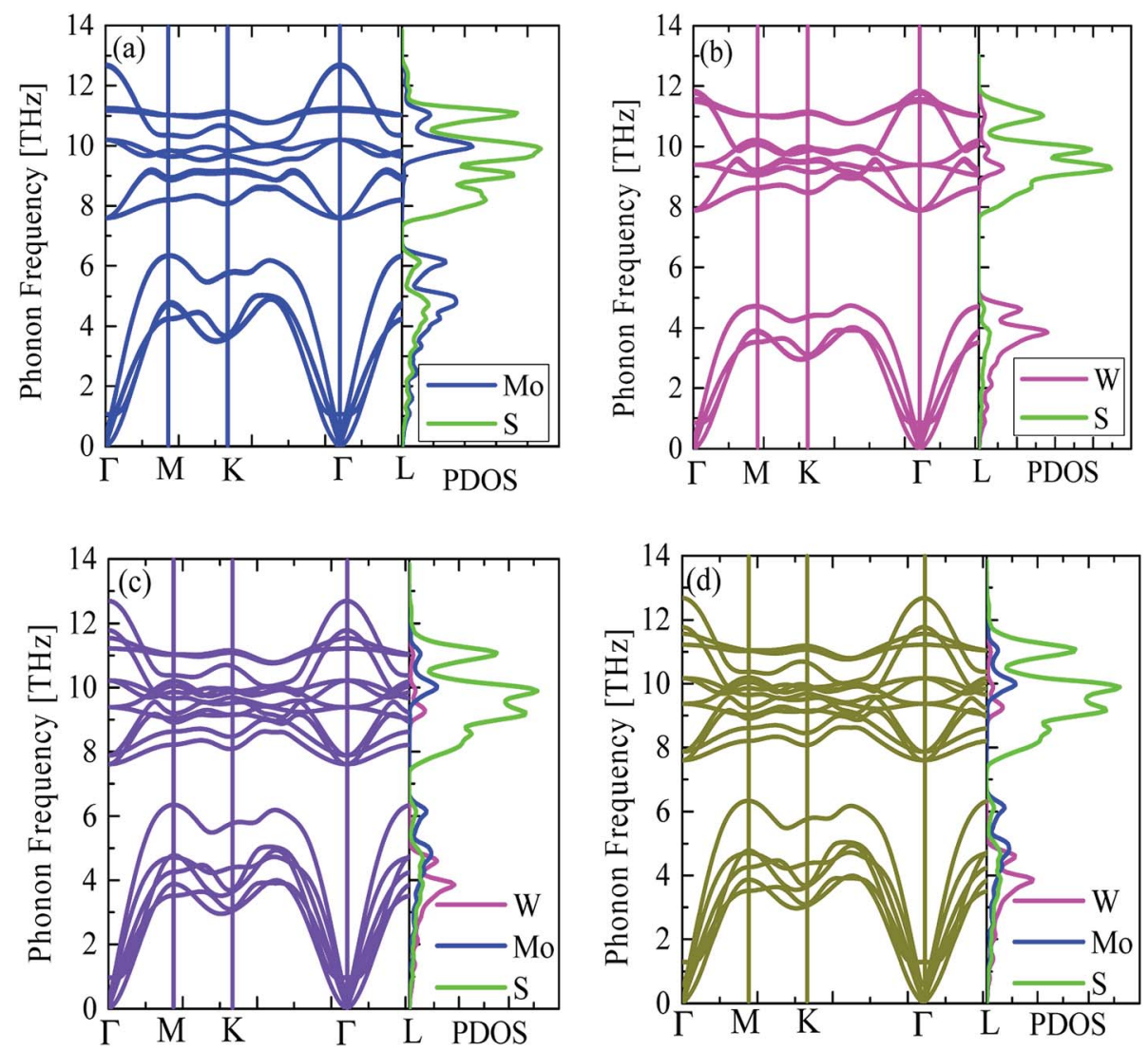

Fig. 2 Phonon dispersion relation and projected phonon density of states (PDOS) of bilayer (a) $\mathrm{MoS}_{2}$, (b) $\mathrm{WS}_{2}$, (c) heterobilayer MoS $/ \mathrm{WS}_{2}$ and (d) superlattice $\mathrm{MoS}_{2} / \mathrm{WS}_{2}$. 
the heterobilayer and superlattice $\mathrm{MoS}_{2} / \mathrm{WS}_{2}$, it finds that their phonon dispersion relations along the lateral direction are the same and interlayer van der Waals forces don't affect the lateral phonon dispersions.

\section{Lateral thermal conductivity}

In Fig. 3(a), the calculated lateral $\kappa$ of bilayer $\mathrm{MoS}_{2}$ and $\mathrm{WS}_{2}$, heterobilayer $\mathrm{MoS}_{2} / \mathrm{WS}_{2}$ and superlattice $\mathrm{MoS}_{2} / \mathrm{WS}_{2}$ are presented and compared with literature experimental data, in order to study the influence of interlayer interactions on lateral thermal transport. By only considering the phonon-phonon interaction, the calculated lateral $\kappa$ of bilayer $\mathrm{MoS}_{2}$ at $300 \mathrm{~K}$ and $500 \mathrm{~K}$ is $61.13 \mathrm{~W} \mathrm{~m}^{-1} \mathrm{~K}^{-1}$ and $36.77 \mathrm{~W} \mathrm{~m}^{-1} \mathrm{~K}^{-1}$, respectively, in good agreement with the measured value of $77 \pm 25 \mathrm{~W} \mathrm{~m}{ }^{-1} \mathrm{~K}^{-1}$ and $29 \pm 10 \mathrm{~W} \mathrm{~m}^{-1} \mathrm{~K}^{-1} \cdot{ }^{17}$ For the bilayer $\mathrm{WS}_{2}$, the calculated lateral $\kappa$ at $300 \mathrm{~K}$ is $87.52 \mathrm{~W} \mathrm{~m}^{-1} \mathrm{~K}^{-1}$, relatively higher than the measured value of $53 \mathrm{~W} \mathrm{~m}{ }^{-1} \mathrm{~K}^{-1} \cdot{ }^{18}$ This difference may be caused by the neglect of electron-phonon interaction (EPI)
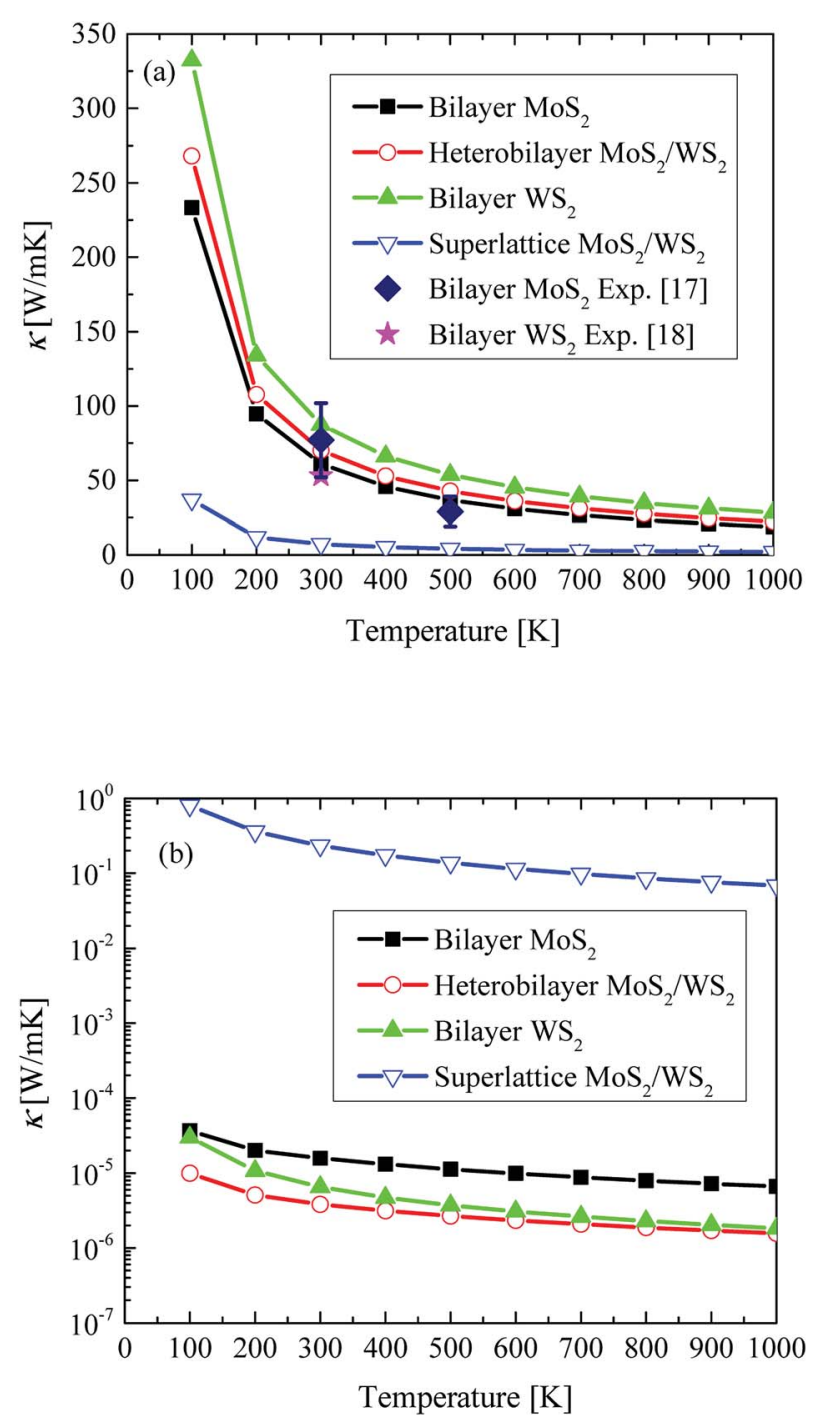

Fig. 3 The calculated (a) lateral and (b) out-of-the-plane $\kappa$ of bilayer $\mathrm{MoS}_{2}$ and $\mathrm{WS}_{2}$, heterobilayer $\mathrm{MoS}_{2} / \mathrm{WS}_{2}$ and superlattice $\mathrm{MoS}_{2} / \mathrm{WS}_{2}$, in comparison with literature data of ref. 17 and 18 . which shows strong effect in the few-layer $\mathrm{WX}_{2}(\mathrm{X}=\mathrm{S}$, Se and Te) crystals. $^{34-36}$ Yet the neglect of EPI calculations which require heavy computing resources does not affect our conclusion about the influence of interlayer interactions on lateral $\kappa$. By comparing the bilayer $\mathrm{MoS}_{2}$ and $\mathrm{WS}_{2}$, heterobilayer $\mathrm{MoS}_{2} /$ $\mathrm{WS}_{2}$, it shows that the lateral $\kappa$ lies between that of bilayer $\mathrm{MoS}_{2}$ and $\mathrm{WS}_{2}$ and preserves the high thermal performance, indicating that the interlayer van der Waals force in the heterobilayer $\mathrm{MoS}_{2} / \mathrm{WS}_{2}$ does not affect the lateral $\kappa$. However, in the superlattice $\mathrm{MoS}_{2} / \mathrm{WS}_{2}$ with alternating $\mathrm{MoS}_{2}$ and $\mathrm{WS}_{2}$ monolayer, the interlayer van der Waals forces has a strong effect and reduces the room-temperature lateral $\kappa$ of heterobilayer $\mathrm{MoS}_{2} /$ $\mathrm{WS}_{2}$ from $70.01 \mathrm{~W} \mathrm{~m}^{-1} \mathrm{~K}^{-1}$ to $7.22 \mathrm{~W} \mathrm{~m}^{-1} \mathrm{~K}^{-1}$ by a factor of 10 .

To interpret such large reduction in lateral $\kappa$, we further compare the calculated $\tau$ and averaged square group velocity $\left(\left\langle v^{2}\right\rangle\right)$ which are the two relevant parameters dominating lateral thermal transport. In Fig. 4(a), by analyzing the cumulative lateral $\kappa$ with phonon frequency, it shows that the propagating phonons mainly (more than 90\%) contributing to the lateral thermal transport lie in the energy range of 1-5 THz. In Fig. 4(b), by comparing $\tau$ in the energy range of 1-5 THz, it observes that the calculated value of superlattice $\mathrm{MoS}_{2} / \mathrm{WS}_{2}$ is one order of magnitude lower than that of bilayer $\mathrm{MoS}_{2}$ and $\mathrm{WS}_{2}$ and heterobilayer $\mathrm{MoS}_{2} / \mathrm{WS}_{2}$. On the other hand, there is little difference in the calculated $\left\langle v^{2}\right\rangle$ along the lateral direction in the energy range of 1-5 THz, as shown in Fig. 4(c). Therefore, it can conclude that the low $\tau$ leads to the greatly reduced lateral $\kappa$ of the superlattice $\mathrm{MoS}_{2} / \mathrm{WS}_{2}$, compared with the heterobilayer. The low $\tau$ means the existence of strong phonon-phonon interaction in the superlattice. To explain it, we first analyze the lattice structures. The structural difference between superlattice and heterobilayer $\mathrm{MoS}_{2} / \mathrm{WS}_{2}$ is that there exists periodically alternating $\mathrm{MoS}_{2}$ and $\mathrm{WS}_{2}$ monolayer in the superlattice, while in the heterobilayer there is a large vacuum layer on top of $\mathrm{MoS}_{2} / \mathrm{WS}_{2}$. The periodically alternating $\mathrm{MoS}_{2}$ and $\mathrm{WS}_{2}$ monolayer introduce large van der Waals forces and induce strong interactions. This will bring large restoring forces for atoms and lead to large group velocity. In Fig. 4(d), the calculated $\left\langle v^{2}\right\rangle$ of superlattice $\mathrm{MoS}_{2} / \mathrm{WS}_{2}$ along the out-of-the-plane direction is eight orders of magnitude larger than that of heterobilayer, which justifies the existence of strong interlayer interactions in the superlattice. Furthermore, the analysis of charge density distribution in Fig. 5 shows that there is a relatively large interlayer charge density along the out-of-the plane direction in the superlattice, while in the heterobilayer there is no interlayer charge density due to the existence of vacuum layer. The existence of interlayer charge density in the superlattice can help explain the strong interlayer interactions. On the other hand, in the alternating $\mathrm{MoS}_{2}$ and $\mathrm{WS}_{2}$ layer of neighboring unit cell in the superlattice, there exists large charge density around Mo and $\mathrm{W}$ atoms, respectively, which would induce the strong interfacial interactions as shown in Fig. 5. The interfacial interactions can enhance the anharmonic scattering among propagating phonons along the plane direction and thus reduce the lateral $\kappa$. To peer more microscopic insights on the interfacial interactions of superlattice $\mathrm{MoS}_{2} / \mathrm{WS}_{2}$, one can analyze the kinetic diffusion and collision process among lateral and 

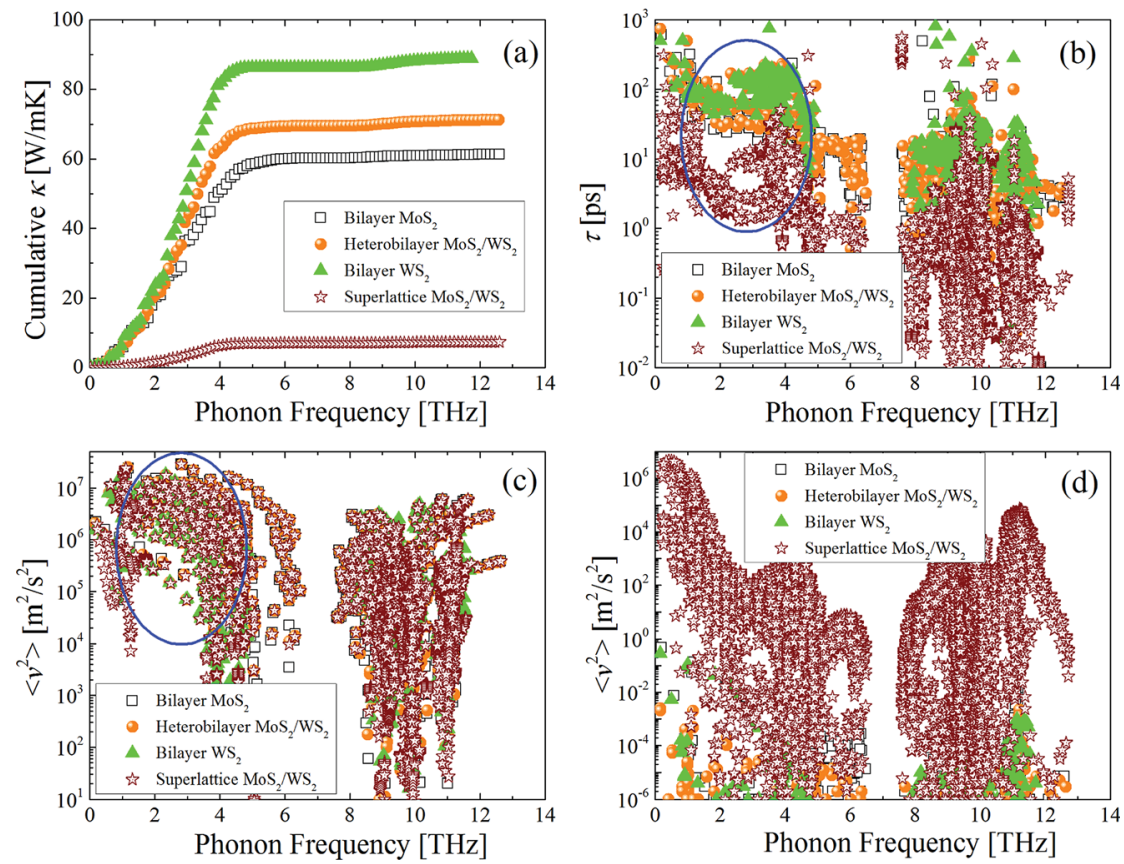

Fig. 4 The (a) cumulative lateral $\kappa_{1}$ (b) phonon relaxation time, (c) and (d) averaged square group velocity along lateral and out-of-the-plane direction, respectively, of bilayer $\mathrm{MOS}_{2}$ and $\mathrm{WS}_{2}$, heterobilayer $\mathrm{MoS}_{2} / \mathrm{WS}_{2}$ and superlattice $\mathrm{MoS}_{2} / \mathrm{WS}_{2}$. The blue ellipse pinpoints the major propagating phonons between 1-5 $\mathrm{THz}$.

flexural phonon modes using the phonon wave-packet dynamics method. ${ }^{37}$ It would require the separation of lateral and flexural phonons but it is beyond the capability of present method.

\section{Out-of-the-plane thermal conductivity}

For the 2D TDMC heterostructures, the interfacial (out-of-the plane) thermal conductance is of great importance for the heat management. In Fig. 3(b), the out-of-the-plane $\kappa$ of bilayer $\mathrm{MoS}_{2}$ and $\mathrm{WS}_{2}$, heterobilayer $\mathrm{MoS}_{2} / \mathrm{WS}_{2}$ and superlattice $\mathrm{MoS}_{2} /$ $\mathrm{WS}_{2}$ are presented. With its nanometer thickness, the roomtemperature out-of-the-plane $\kappa$ of bilayer $\mathrm{MoS}_{2}$ and $\mathrm{WS}_{2}$ and heterobilayer $\mathrm{MoS}_{2} / \mathrm{WS}_{2}$ is limited to be $1.58 \times 10^{-5} \mathrm{~W} \mathrm{~m}^{-1} \mathrm{~K}^{-1}$, $6.52 \times 10^{-6} \mathrm{~W} \mathrm{~m}^{-1} \mathrm{~K}^{-1}$ and $3.86 \times 10^{-6} \mathrm{~W} \mathrm{~m}^{-1} \mathrm{~K}^{-1}$, respectively. It shows that the vertically stacking $\mathrm{MoS}_{2}$ and $\mathrm{WS}_{2}$ monolayer do not influence the interfacial thermal (a)

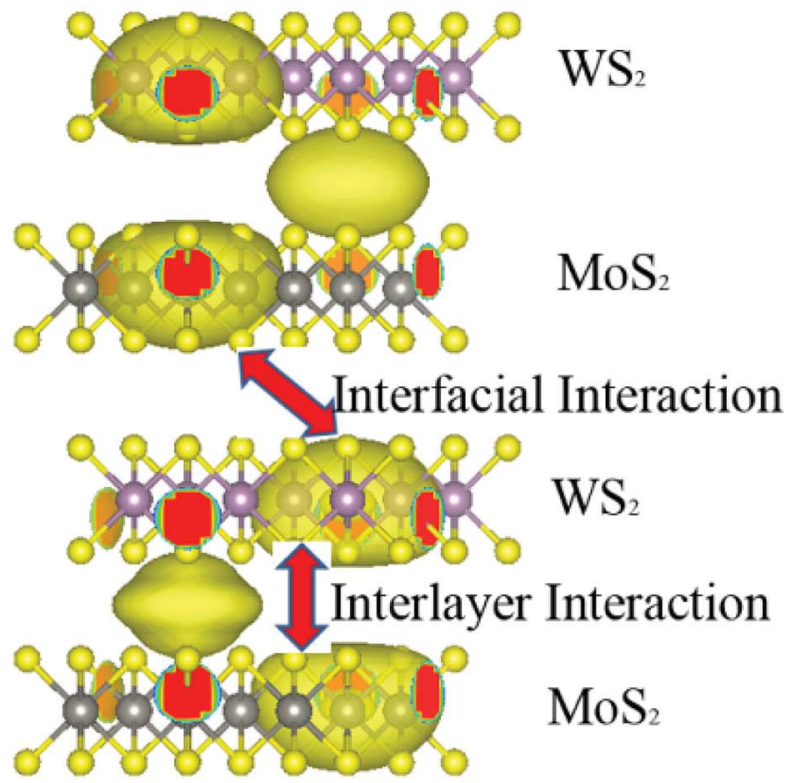

(b)
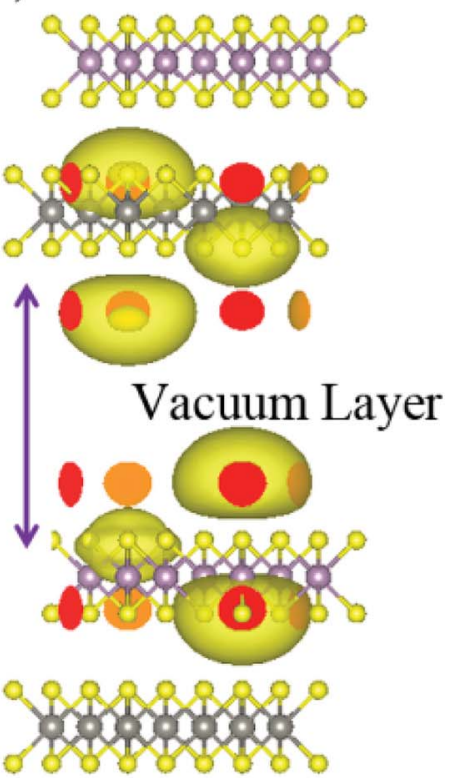

Fig. 5 Charge density distribution of (a) superlattice and (b) heterobilayer $\mathrm{MoS}_{2} / \mathrm{WS}_{2}$. 
conductance. By periodically alternating $\mathrm{MoS}_{2}$ and $\mathrm{WS}_{2}$ monolayer with strong interlayer interactions, the room-temperature out-of-the-plane $\kappa$ of superlattice $\mathrm{MoS}_{2} / \mathrm{WS}_{2}$ is increased to be $0.23 \mathrm{~W} \mathrm{~m}^{-1} \mathrm{~K}^{-1}$ and 5 orders of magnitude larger than that of heterobilayer.

\section{Conclusions}

In summary, we have calculated four prototype TDMC heterostructures (bilayer $\mathrm{MoS}_{2}$ and $\mathrm{WS}_{2}$, heterobilayer $\mathrm{MoS}_{2} / \mathrm{WS}_{2}$ and superlattice $\mathrm{MoS}_{2} / \mathrm{WS}_{2}$ ) and investigated their lateral and out-ofthe-plane $\kappa$ from first-principles. By analyzing the anharmonic lattice dynamics coupled with solving the phonon Boltzmann transport equation, the calculated lateral $\kappa$ of bilayer $\mathrm{MoS}_{2}$ and $\mathrm{WS}_{2}$ at $300 \mathrm{~K}$ is $61.13 \mathrm{~W} \mathrm{~m}^{-1} \mathrm{~K}^{-1}$ and $87.52 \mathrm{~W} \mathrm{~m}^{-1} \mathrm{~K}^{-1}$, respectively, in reasonable agreement with experimental data of $77 \pm 25 \mathrm{~W} \mathrm{~m}^{-1} \mathrm{~K}^{-1}$ and $53 \mathrm{~W} \mathrm{~m} \mathrm{~m}^{-1} \mathrm{~K}^{-1}$. By vertically stacking the $\mathrm{MoS}_{2}$ and $\mathrm{WS}_{2}$ monolayer together and applying a nanometersized vacuum layer on its top, the heterobilayer $\mathrm{MoS}_{2} / \mathrm{WS}_{2}$ forms and it preserves the high lateral $\kappa$ of $70.01 \mathrm{~W} \mathrm{~m}^{-1} \mathrm{~K}^{-1}$ at $300 \mathrm{~K}$, which indicates that the weak interlayer interactions do not affect the lateral thermal transport. Moreover, the coupled quantum states of vertically stacking $\mathrm{MoS}_{2}$ and $\mathrm{WS}_{2}$ monolayer do not influence the out-of-the-plane $\kappa$. For the superlattice composed of periodically alternating $\mathrm{MoS}_{2}$ and $\mathrm{WS}_{2}$ monolayer, the charge density distribution analysis shows that there exist strong interlayer and interfacial interactions. Consequently, the interfacial anharmonic phonon scattering is enhanced and phonon relaxation time is greatly decreased for the propagating phonons, leading to a significant reduction of lateral $\kappa$ from $70.01 \mathrm{~W} \mathrm{~m}^{-1} \mathrm{~K}^{-1}$ to $7.22 \mathrm{~W} \mathrm{~m}^{-1} \mathrm{~K}^{-1}$ almost by a factor of 10 . This work shows that the strong interfacial interactions indeed influence the lateral thermal transport in the superlattice $\mathrm{MoS}_{2}$ / $\mathrm{WS}_{2}$. The physical insights obtained from this work can help predict the thermal transport in other TDMC heterostructures and advance the thermal management in their applications in nanoelectronics and optoelectronics.

\section{Conflicts of interest}

There are no conflicts to declare.

\section{Acknowledgements}

J.-Y. Y. is grateful for the financial support by the Excellence Initiative of the German Federal and State Governments (Project No. StUpPD_268-17) and W. Zhang thanks the support by the Shandong Provincial Natural Science Foundation, China (No. ZR2017BEE051). We gratefully acknowledge the computing time granted by the John von Neumann Institute for Computing (NIC) and provided on the supercomputer JURECA at Forschungszentrum Jülich, Germany (Project ID: JHPC51).

\section{References}

1 P. Rivera, H. Yu, K. L. Seyler, N. P. Wilson, W. Yao and X. Xu, Nat. Nanotechnol., 2018, 13, 1004-1015.
2 C. Jin, E. Y. Ma, O. Karni, E. C. Regan, F. Wang and T. F. Heinz, Nat. Nanotechnol., 2018, 13, 994-1003.

3 H. Yu, X. Cui, X. Xu and W. Yao, Natl. Sci. Rev., 2015, 2, 57-70.

4 A. M. Jones, H. Yu, N. J. Ghimire, S. Wu, G. Aivazian, J. S. Ross, B. Zhao, J. Yan, D. G. Mandrus, D. Xiao, W. Yao and X. Xu, Nat. Nanotechnol., 2013, 8, 634.

5 D. Xiao, G.-B. Liu, W. Feng, X. Xu and W. Yao, Phys. Rev. Lett., 2012, 108, 196802.

6 W. Zhao, Z. Ghorannevis, K. K. Amara, J. R. Pang, M. Toh, X. Zhang, C. Kloc, P. H. Tan and G. Eda, Nanoscale, 2013, 5, 9677-9683.

7 L. Yuan and L. Huang, Nanoscale, 2015, 7, 7402-7408.

8 B. Hunt, J. D. Sanchez-Yamagishi, A. F. Young, M. Yankowitz, B. J. LeRoy, K. Watanabe, T. Taniguchi, P. Moon, M. Koshino, P. Jarillo-Herrero and R. C. Ashoori, Science, 2013, 340, 14271430.

9 C. R. Dean, L. Wang, P. Maher, C. Forsythe, F. Ghahari, Y. Gao, J. Katoch, M. Ishigami, P. Moon, M. Koshino, T. Taniguchi, K. Watanabe, K. L. Shepard, J. Hone and P. Kim, Nature, 2013, 497, 598.

10 Y. Cao, V. Fatemi, S. Fang, K. Watanabe, T. Taniguchi, E. Kaxiras and P. Jarillo-Herrero, Nature, 2018, 556, 43.

11 K. S. Novoselov, A. Mishchenko, A. Carvalho and A. H. Castro Neto, Science, 2016, 353, aac9439.

12 Z. Zhang, P. Chen, X. Duan, K. Zang, J. Luo and X. Duan, Science, 2017, 357, 788-792.

13 S. Manzeli, D. Ovchinnikov, D. Pasquier, O. V. Yazyev and A. Kis, Nat. Rev. Mater., 2017, 2, 17033.

14 K. Chen, X. Wan, J. Wen, W. Xie, Z. Kang, X. Zeng, H. Chen and J.-B. Xu, ACS Nano, 2015, 9, 9868-9876.

15 W. Aggoune, C. Cocchi, D. Nabok, K. Rezouali, M. Akli Belkhir and C. Draxl, J. Phys. Chem. Lett., 2017, 8, 1464-1471.

16 C. Chen, J. Avila, S. Wang, Y. Wang, M. Mucha-Kruczyński,

C. Shen, R. Yang, B. Nosarzewski, T. P. Devereaux, G. Zhang and M. C. Asensio, Nano Lett., 2018, 18, 1082-1087.

17 X. Zhang, D. Sun, Y. Li, G.-H. Lee, X. Cui, D. Chenet, Y. You, T. F. Heinz and J. C. Hone, ACS Appl. Mater. Interfaces, 2015, 7, 25923-25929.

18 N. Peimyoo, J. Shang, W. Yang, Y. Wang, C. Cong and T. Yu, Nano Res., 2015, 8, 1210-1221.

19 J. Zhang, Y. Hong, X. Wang, Y. Yue, D. Xie, J. Jiang, Y. Xiong and P. Li, J. Phys. Chem. C, 2017, 121, 10336-10344.

20 J.-Y. Yang, L. Cheng and M. Hu, Appl. Phys. Lett., 2017, 111, 242101.

21 J.-Y. Yang, S.-Y. Yue and M. Hu, Phys. Rev. B, 2016, 94, 235153.

22 J. M. Ziman, Electrons and phonons: the theory of transport phenomena in solids, Oxford University Press, 1960.

23 D. A. Broido, A. Ward and N. Mingo, Phys. Rev. B: Condens. Matter Mater. Phys., 2005, 72, 014308.

24 K. Esfarjani, G. Chen and H. T. Stokes, Phys. Rev. B: Condens. Matter Mater. Phys., 2011, 84, 085204.

25 W. Li, J. Carrete, N. A. Katcho and N. Mingo, Comput. Phys. Commun., 2014, 185, 1747-1758.

26 L. Lindsay, D. A. Broido and T. L. Reinecke, Phys. Rev. B: Condens. Matter Mater. Phys., 2013, 87, 165201.

27 A. Togo and I. Tanaka, Scr. Mater., 2015, 108, 1-5. 
28 G. Kresse and J. Furthmüller, Phys. Rev. B: Condens. Matter Mater. Phys., 1996, 54, 11169-11186.

29 P. E. Blöchl, Phys. Rev. B: Condens. Matter Mater. Phys., 1994, 50, 17953-17979.

30 J. P. Perdew, Phys. Rev. B: Condens. Matter Mater. Phys., 1986, 33, 8822.

31 J. P. Perdew, K. Burke and M. Ernzerhof, Phys. Rev. Lett., 1996, 77, 3865-3868.

32 K. Lee, É. D. Murray, L. Kong, B. I. Lundqvist and D. C. Langreth, Phys. Rev. B: Condens. Matter Mater. Phys., 2010, 82, 081101.

33 H. J. Monkhorst and J. D. Pack, Phys. Rev. B: Condens. Matter Mater. Phys., 1976, 13, 5188-5192.

34 N. F. Hinsche, A. S. Ngankeu, K. Guilloy, S. K. Mahatha, A. Grubišić Čabo, M. Bianchi, M. Dendzik, C. E. Sanders,
J. A. Miwa, H. Bana, E. Travaglia, P. Lacovig, L. Bignardi, R. Larciprete, A. Baraldi, S. Lizzit, K. S. Thygesen and P. Hofmann, Phys. Rev. B, 2017, 96, 121402.

35 A. P. S. Gaur, S. Sahoo, J. F. Scott and R. S. Katiyar, J. Phys. Chem. C, 2015, 119, 5146-5151.

36 I. Niehues, R. Schmidt, M. Drüppel, P. Marauhn, D. Christiansen, M. Selig, G. Berghäuser, D. Wigger, R. Schneider, L. Braasch, R. Koch, A. Castellanos-Gomez, T. Kuhn, A. Knorr, E. Malic, M. Rohlfing, S. Michaelis de Vasconcellos and R. Bratschitsch, Nano Lett., 2018, 18, 1751-1757.

37 P. K. Schelling, S. R. Phillpot and P. Keblinski, Appl. Phys. Lett., 2002, 80, 2484-2486. 